\title{
Use of gene expression studies to investigate the human immunological response to malaria infection
}

Susanne H. Hodgson ${ }^{1,2^{*}} \mathbb{0}$, Julius Muller ${ }^{1}$, Helen E. Lockstone ${ }^{3}$, Adrian V. S. Hill ${ }^{1,3}$, Kevin Marsh $^{4}$, Simon J. Draper ${ }^{1}$ and Julian C. Knight ${ }^{3}$

\begin{abstract}
Background: Transcriptional profiling of the human immune response to malaria has been used to identify diagnostic markers, understand the pathogenicity of severe disease and dissect the mechanisms of naturally acquired immunity (NAI). However, interpreting this body of work is difficult given considerable variation in study design, definition of disease, patient selection and methodology employed. This work details a comprehensive review of gene expression profiling (GEP) of the human immune response to malaria to determine how this technology has been applied to date, instances where this has advanced understanding of NAl and the extent of variability in methodology between studies to allow informed comparison of data and interpretation of results.
\end{abstract}

Methods: Datasets from the gene expression omnibus (GEO) including the search terms; 'plasmodium' or 'malaria' or 'sporozoite' or 'merozoite' or 'gametocyte' and 'Homo sapiens' were identified and publications analysed. Datasets of gene expression changes in relation to malaria vaccines were excluded.

Results: Twenty-three GEO datasets and 25 related publications were included in the final review. All datasets related to Plasmodium falciparum infection, except two that related to Plasmodium vivax infection. The majority of datasets included samples from individuals infected with malaria 'naturally' in the field $(n=13,57 \%)$, however some related to controlled human malaria infection (CHMI) studies $(n=6,26 \%)$, or cells stimulated with Plasmodium in vitro $(n=6,26 \%)$. The majority of studies examined gene expression changes relating to the blood stage of the parasite. Significant heterogeneity between datasets was identified in terms of study design, sample type, platform used and method of analysis. Seven datasets specifically investigated transcriptional changes associated with NAl to malaria, with evidence supporting suppression of the innate pro-inflammatory response as an important mechanism for this in the majority of these studies. However, further interpretation of this body of work was limited by heterogeneity between studies and small sample sizes.

Conclusions: GEP in malaria is a potentially powerful tool, but to date studies have been hypothesis generating with small sample sizes and widely varying methodology. As CHMl studies are increasingly performed in endemic settings, there will be growing opportunity to use GEP to understand detailed time-course changes in host response and understand in greater detail the mechanisms of NAI.

Keywords: Plasmodium falciparum, Gene expression, Malaria, Immunity

*Correspondence: susannesheehy@doctors.org.uk

1 The Jenner Institute, University of Oxford, Old Road Campus Road Building, Off Roosevelt Drive, Oxford OX3 7DQ, UK

Full list of author information is available at the end of the article

\section{Background}

Malaria, caused by infection with parasites of the genus Plasmodium, remains a significant public health concern [1]. Despite a vaccine in pilot implementation trials [2] and widespread application of control measures [3], the disease is still responsible for a huge burden of mortality 
and morbidity worldwide and a concerning increase in incidence has been seen in previously well-controlled areas [3].

With repeated exposure to infection, individuals in malaria-endemic regions develop naturally acquired immunity (NAI), first to the most severe clinical forms, such as cerebral malaria and then more slowly to infection itself [1]. Although the role of antibodies in controlling parasite density, symptomatology and severity of disease is well established $[4,5]$, less is known about mechanism in terms of the role of the innate and cellular immune responses [6]. Increased understanding of the immune response to malaria, in particular those that mediate NAI, could aid identification of diagnostic and prognostic markers, inform vaccine development and assist with the identification of treatment strategies to modify the immunological mechanisms mediating severe pathology [1].

Transcriptomics, which allows the expression of thousands of genes to be assessed in parallel for a single RNA sample, is an exciting, expanding area of research with vast potential application in the field of infection [7]. Facilitating a systems biology approach, gene expression data from high-throughput technologies (such as microarrays [8] and next generation sequencing enabling RNA sequencing for bulk cell populations and at singlecell resolution $[9,10])$ can allow greater understanding of individuals' response to infection. To date, expression data have been used to dissect mechanisms of vaccine immunogenicity [11], inform the design of new vaccines $[12,13]$, predict response to infection and outcome [14, 15], characterize and improve understanding of sepsis [16], and offer a novel approach to the diagnosis of infectious pathogens [17-19] together with RNA expression in the pathogen [20].

Given the limited understanding of the mechanisms of NAI to malaria from traditional immunological studies, a systems approach characterizing the gene expression patterns associated with infection could provide novel and valuable insights [21, 22]. Transcriptional profiling of the immune response to malaria in humans to date has sought to identify markers to aid diagnosis [23], to understand the pathogenicity of severe disease [24] and dissect the mechanisms of NAI $[25,26]$. However, interpreting this body of work is difficult given considerable variation in study design, definition of disease, patient selection and methodology employed.

This review outlines a comprehensive analysis of all GEP studies of the human immune response to malaria with two aims: (i) to understand the application of this technology to date, in particular how these studies have informed understanding of NAI; and (ii) to determine the extent of variability in methodology between studies to allow informed comparison of data and interpretation of results.

\section{Methods}

A search of Gene Expression Omnibus (GEO) [27] for datasets including the search terms; 'plasmodium' or 'malaria' or 'sporozoite' or 'merozoite' or 'gametocyte' and 'Homo sapiens' was performed on 10th September 2019. Each of these datasets were examined and those not relating to the human immune response to malaria infection or using the Homo sapiens platform excluded. Of note, datasets of gene expression changes in relation to malaria vaccines were excluded.

\section{Results}

\section{Studies identified}

The search identified 30 GEO datasets. Seven of these datasets were excluded, as published analyses were unavailable. Twenty-three datasets and 25 related publications were therefore included in the final review (Table 1 and Additional file 1: Figure S1). All datasets related to Plasmodium falciparum infection except two that related to Plasmodium vivax infection (Table 1). The majority of datasets included samples from individuals infected with malaria 'naturally' in the field $(\mathrm{n}=13,57 \%)$, however some related to controlled human malaria infection (CHMI) studies $(\mathrm{n}=6,26 \%)$, or cells stimulated with Plasmodium in vitro $(n=6,26 \%)$. Studies included samples from individuals with a wide range of ages (from 2 months-varying ages of adulthood) with differing degrees of prior exposure and, therefore, NAI to malaria. Samples were often collected as part of wider immunoepidemiological studies or vaccine trials, leading to variation in study design and sampling intervals.

\section{Review of methodological approaches}

Significant heterogeneity in the datasets was found in terms of study design, sample type, platform used and method of analysis (Tables 1,2 and Fig. 1), making direct comparison of results between studies difficult. Most datasets were generated from whole blood samples $(\mathrm{n}=11,48 \%)$, however some used PBMCs $(\mathrm{n}=3,13 \%)$ or individual tissue or cells types $(\mathrm{n}=8,35 \%)$ (Table 1$)$. For the majority of studies, expression profiling was performed by array $(n=16,70 \%)$, with others using high throughput sequencing $(n=6,26 \%)$ or RT-qPCR [28] $(\mathrm{n}=1,4 \%)$ (Table 1). There was heterogeneity in data generation between studies with variation in methods used for normalization of data and adjustment for co-variables (Table 2). Thresholds for significance varied considerably and not all studies applied corrections for multiple testing. Choice of database used for gene ontology analysis 


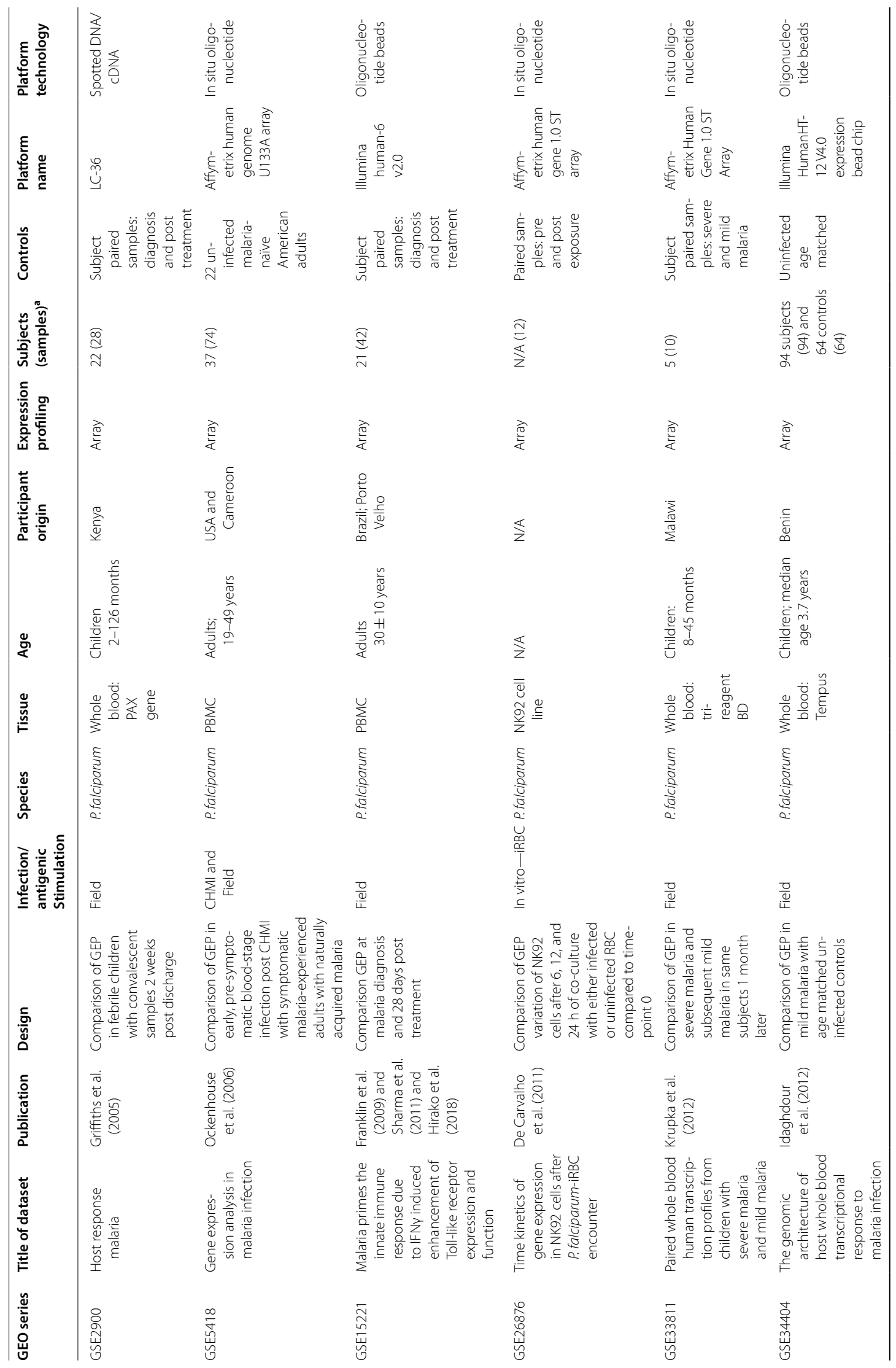




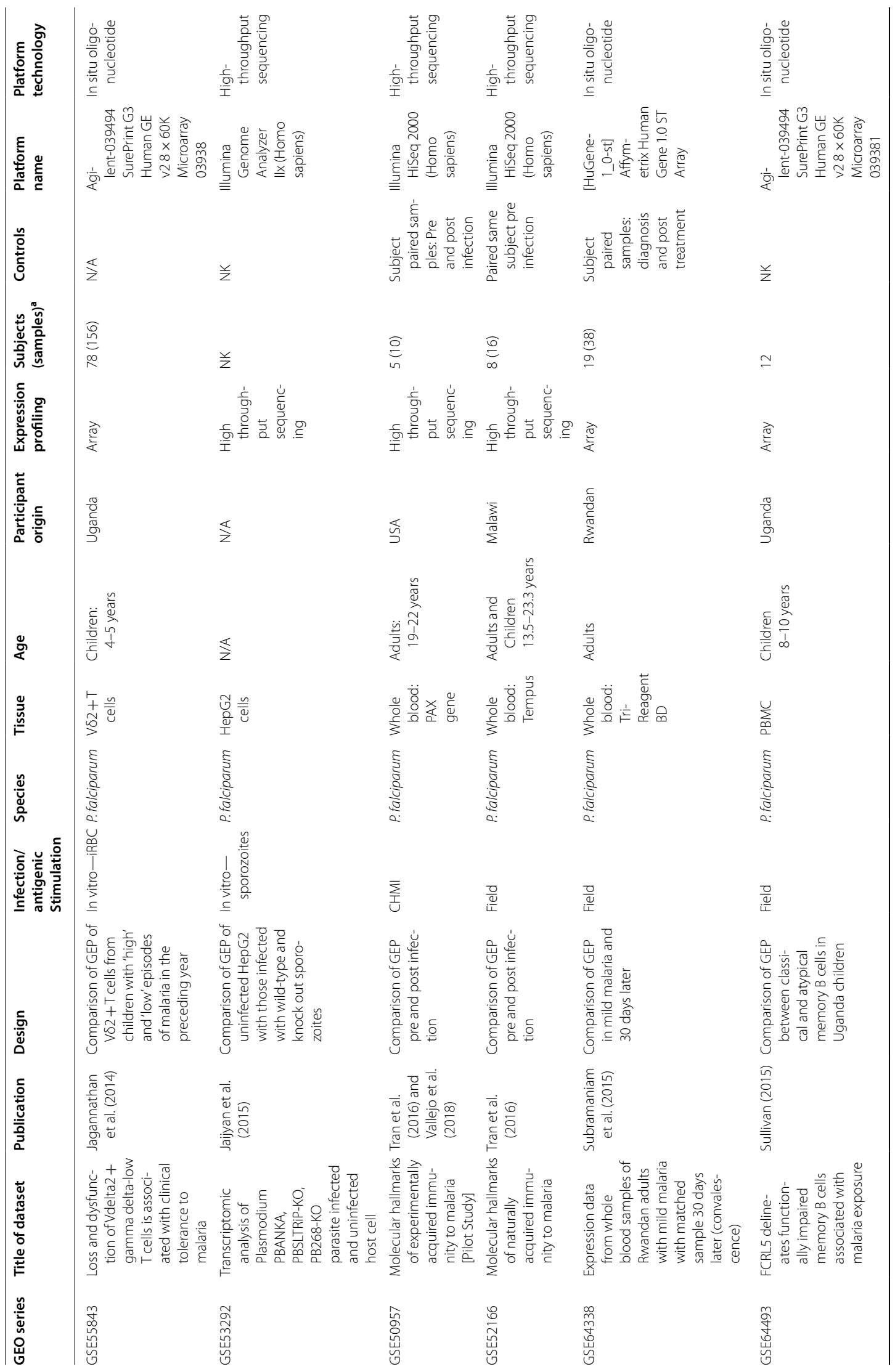




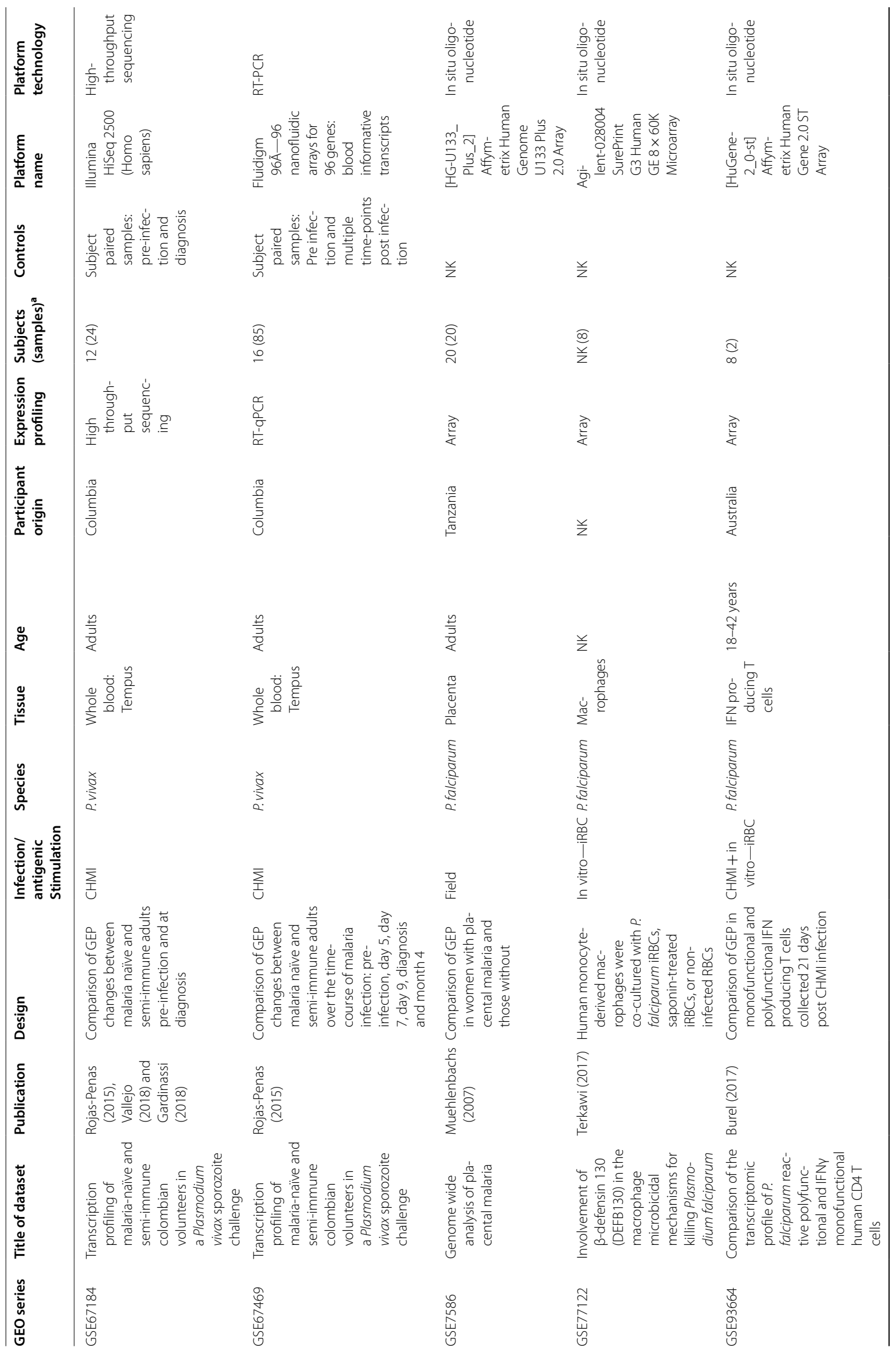




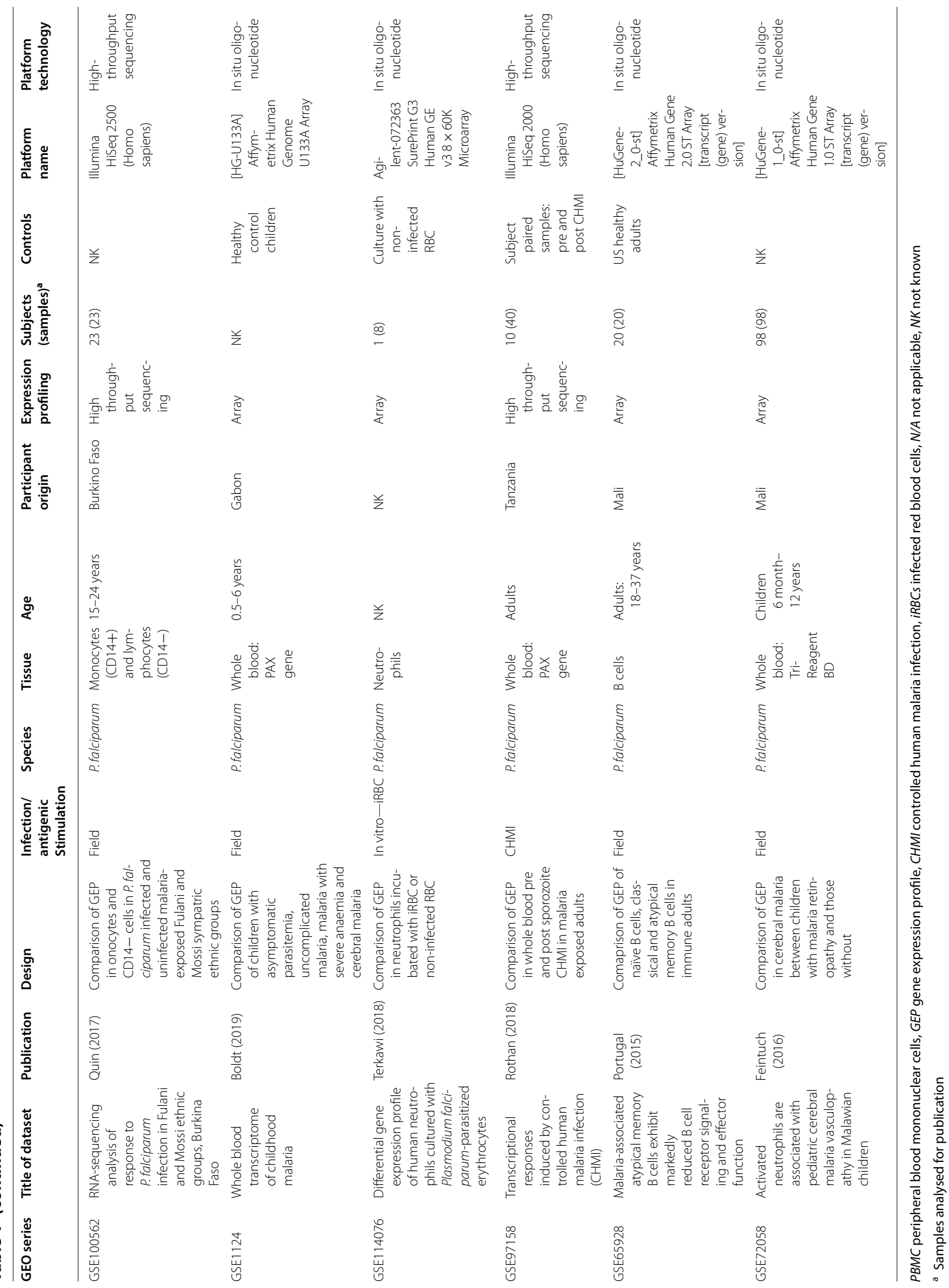




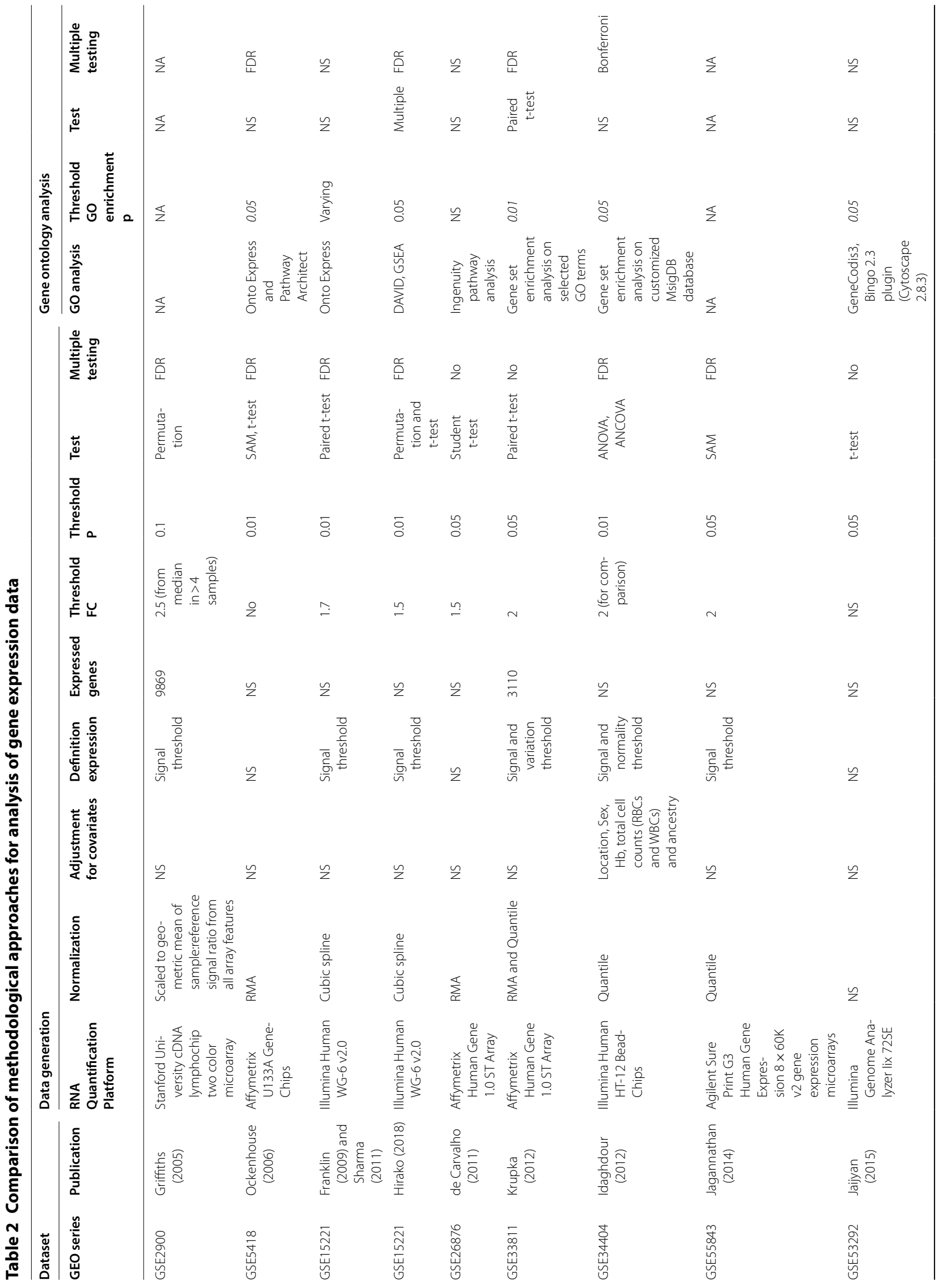




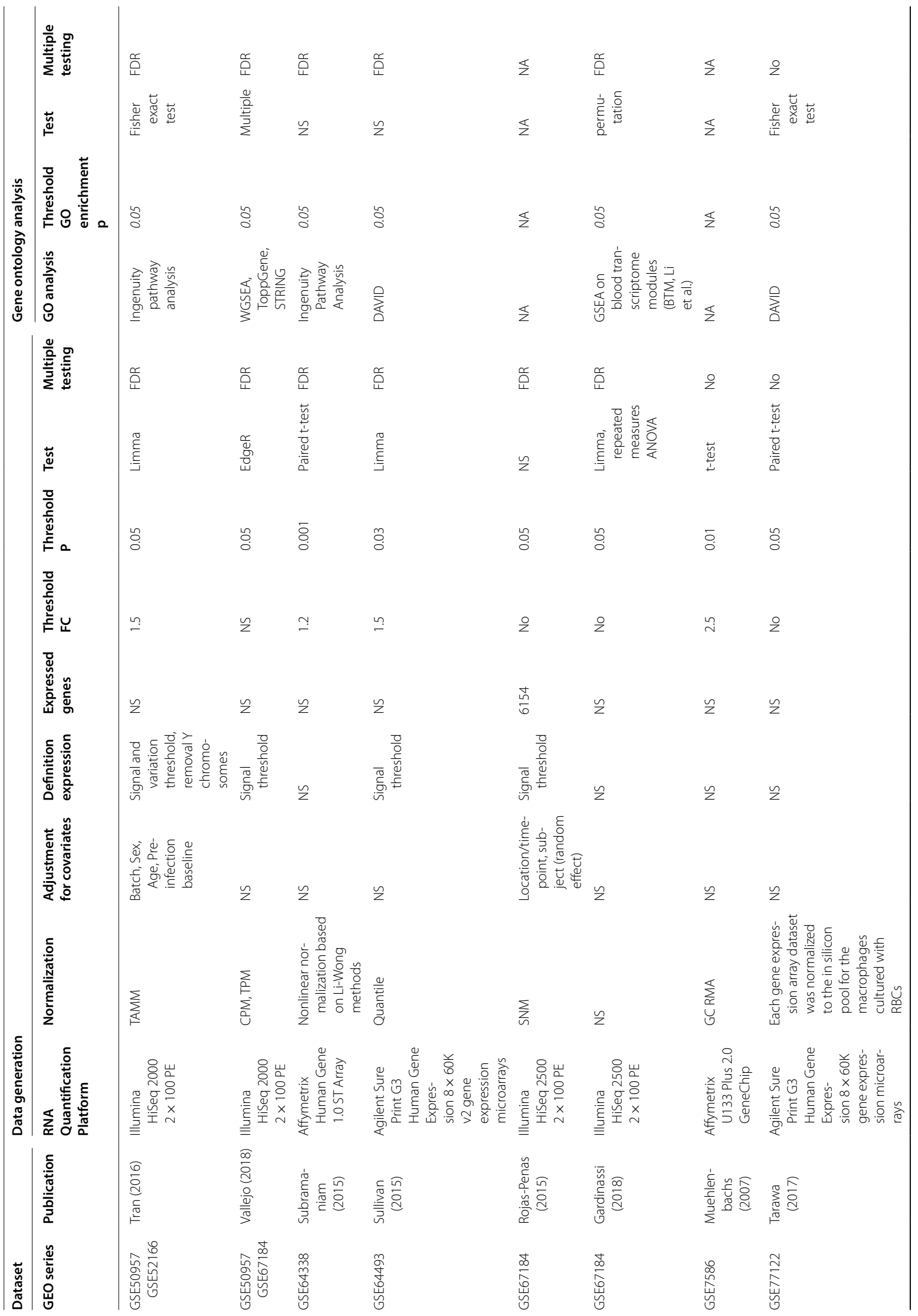




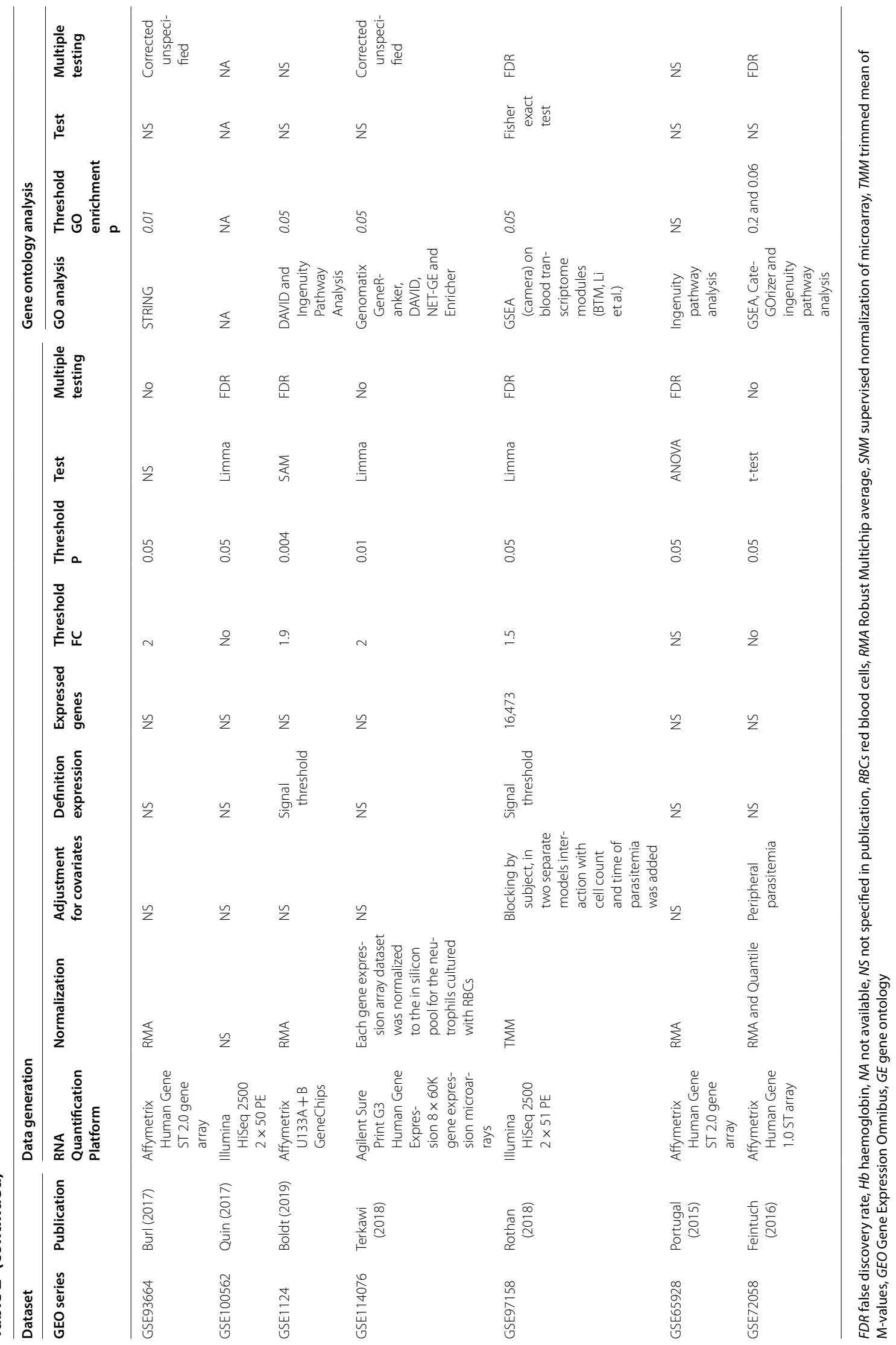


also varied and there was variable, often incomplete reporting of analysis methods used (Table 2).

\section{Transcriptional insights into the immune response to malaria infection}

Seven datasets provided insight into the transcriptional changes associated with NAI to malaria (Table 3) [2426, 28-31]. However, given the difficulty in defining or quantifying NAI for an individual, studies varied in their approach, choosing to examine GEPs in settings of varying history of prior exposure to malaria $[25,26,28,29]$, symptomatology during infection [25] or severity of disease $[24,32]$. All studies examining NAI included small numbers of subjects and all deployed different experimental designs (Table 3).
The findings from a number of studies supported a dampening of the innate pro-inflammatory immune response as a mechanism underpinning NAI [24-26, 33] although this finding was not observed in all studies [28, $29,31]$.

One study by Franklin et al. provided evidence of 'proinflammatory priming' of the innate immune system in acute malaria infection [34]. Comparison of GEP in Brazilian adults presenting with uncomplicated malaria with paired convalescent samples showed an increase in expression in genes involved in TLR signalling pathways supporting a role for TLR hyper responsiveness in the pathology of malaria infection [34, 35].

Quin et al. sought to use RNA sequencing to elucidate the mechanism driving lower infection rates, lower
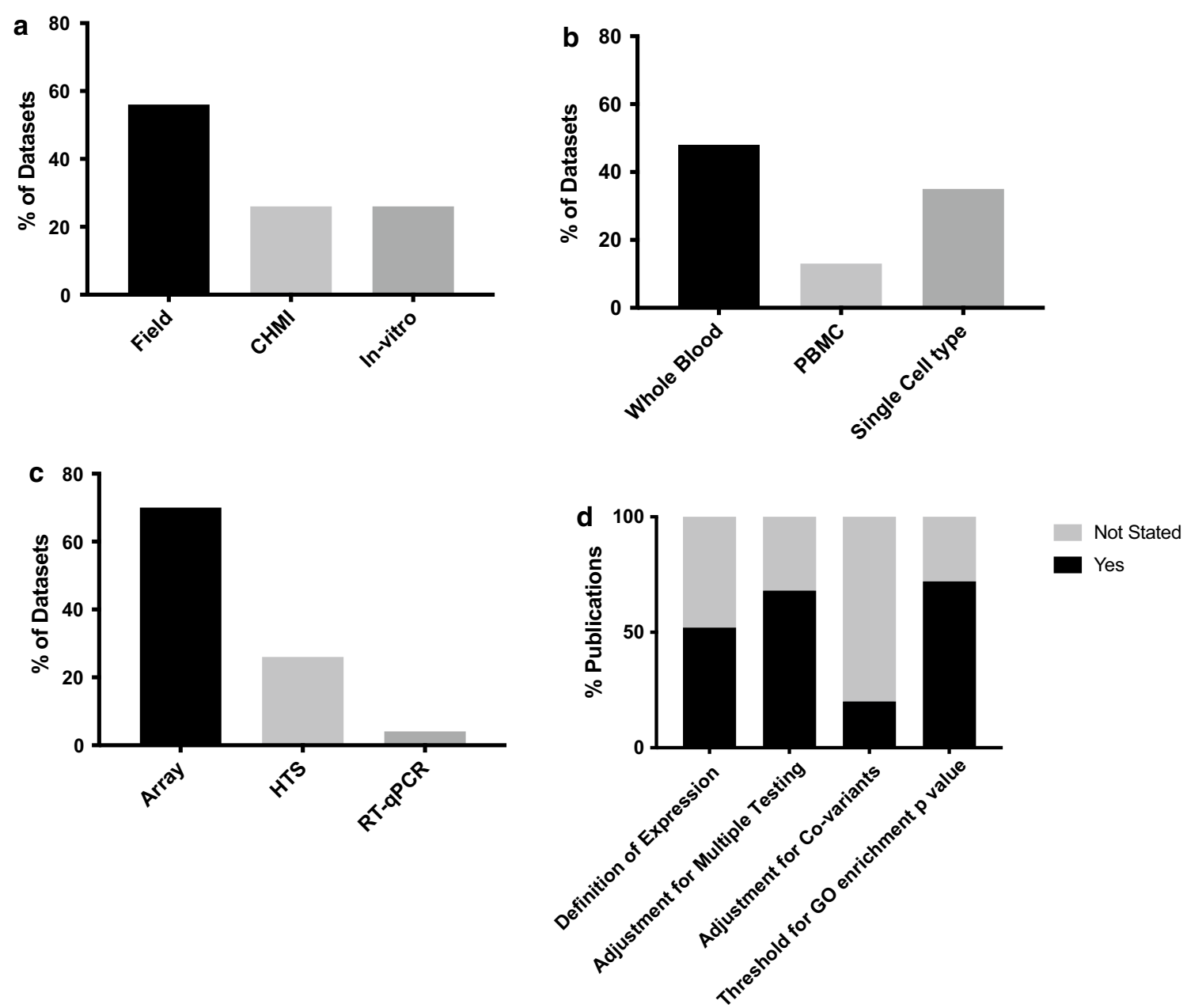

Fig. 1 Comparison of key methodological variables between datasets or publications. a Antigenic stimulation; CHMl controlled human malaria infection, 'field' infection naturally by mosquito bite, 'in-vitro' in vitro stimulation by sporozoites or infected red blood cells. Some datasets employed more than one method of antigenic stimulation. $\mathbf{b}$ Tissue type analysed; PBMC peripheral blood mononuclear cells. $\mathbf{c}$ Expression profiling method: HTS high throughput sequencing. $\mathbf{d}$ Manipulation of data, go gene ontology 


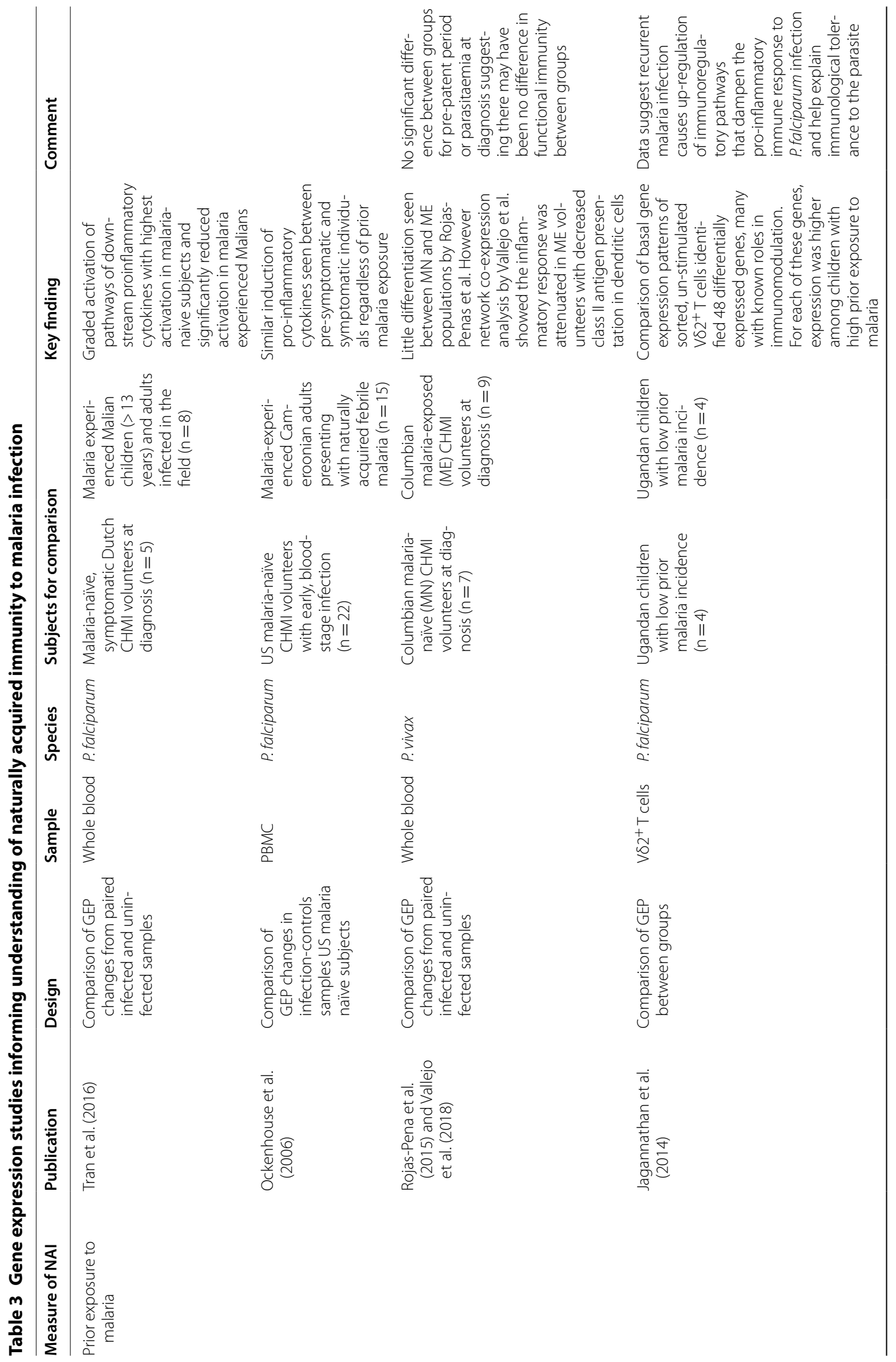




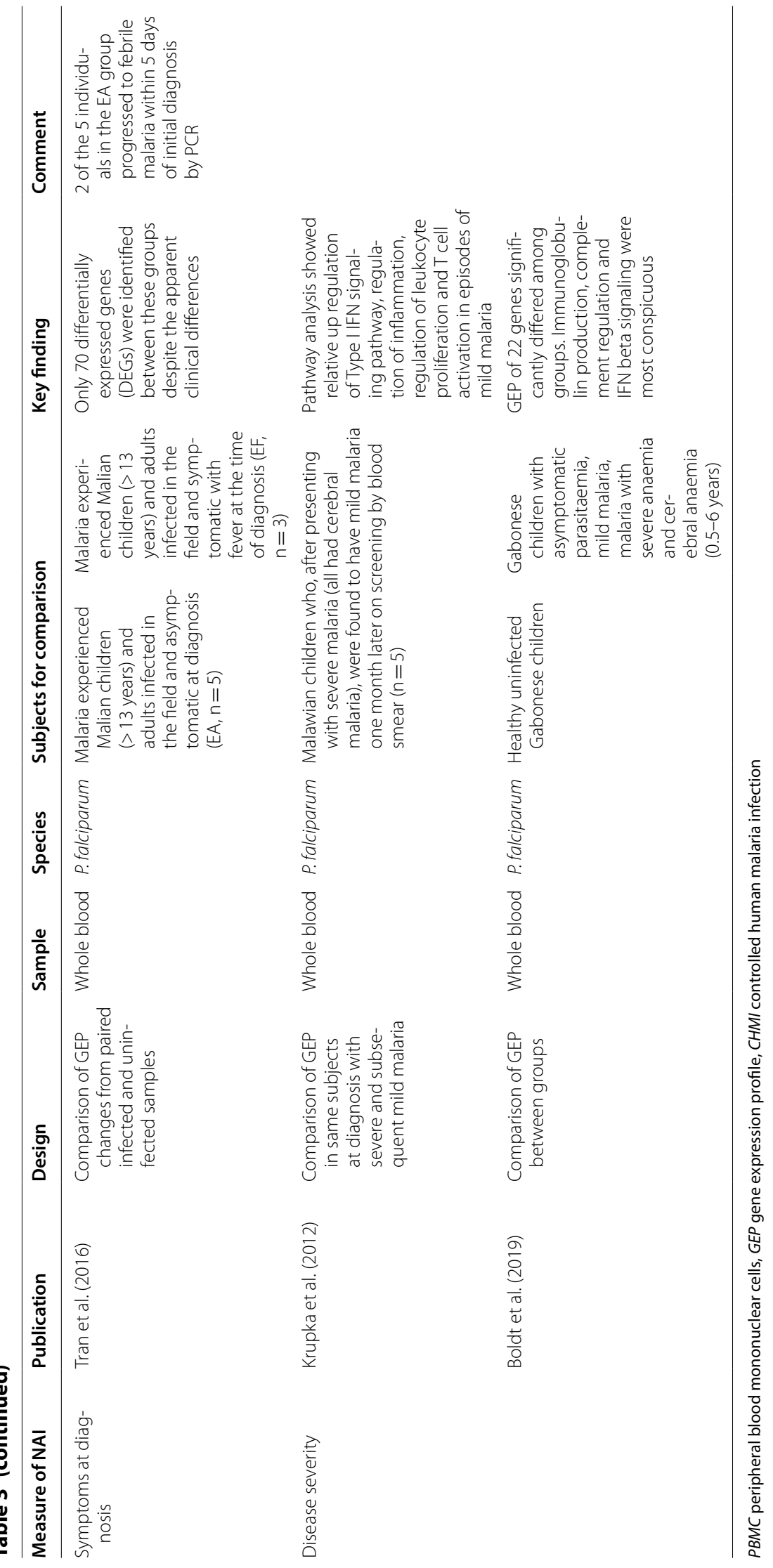


parasite densities and fewer symptomatic cases of $P$. falciparum in the population of Fulani compared to other sympatric ethnic groups [33]. Comparison of the GEP of monocytes from infected and uninfected Fulani and Mossi adults showed a marked difference, with a significantly greater number of differentially expressed (DE) genes in infected Fulani compared to infected Mossi participants (1239 versus 3 DE genes respectively). Pathway analysis showed that infected Fulani, but not infected Mossi, individuals demonstrated a marked reduction in expression of inflammasome pathway components, suggesting a blunting of the innate pro-inflammatory immune response post-infection could explain the differences in susceptibility.

Another study sought to examine the genetic basis of gene expression variation in malaria [36]. Idaghdour et al. compared GEP in children diagnosed with uncomplicated malaria $(\mathrm{n}=94)$ in Benin with age matched controls $(n=64)$ [36] and performed a genome wide association test of transcript abundance. Testing for genotype-by-infection interactions demonstrated the existence of genome wide significant interactions and other genes subject to interaction effects beneath genomewide significance but still likely to have important roles in modulating the course of infection. These interactions affected the complement system, antigen processing and presentation and $\mathrm{T}$ cell activation [36].

In work to identify a transcriptional signature to distinguish acute malaria from other febrile illnesses, Griffiths et al. compared the GEP of twenty-two Kenyan children admitted with febrile illnesses (fifteen of which had malaria infection alone) with six convalescent samples collected 2 weeks post discharge [23]. Two main GEPs relating to neutrophil and erythroid activity were shown to differentiate acutely ill and convalescent children, with significantly higher expression of genes in the neutrophil-related gene region in subjects with bacterial infections and significantly higher expression of genes related to lymphocyte and $\mathrm{T}$ cell activation in subjects with malaria. The authors also identified two gene profiles whose expression intensity correlated with host parasitaemia.

Only two datasets included gene expression changes following $P$. vivax infection [28, 30,37]. Rojas-Penas et al. interrogated GEP changes in malaria naïve $(\mathrm{MN})$ and malaria-exposed (ME) Columbian volunteers following infection with $P$. vivax in a CHMI setting [28]. Significant GEP changes were consistent with time-point rather than prior malaria exposure, with a decline in innate immune signalling and neutrophil number (in contrast to strong up regulation of the same genes reported by Igadour et al. [36]) and an increase in interferon induction seen at diagnosis. No significant GEP changes were noted at other time points, including those relating to the liver stage of infection. Further analysis of this dataset by Vallejo et al. using network co-expression analysis showed that while $P$. vivax infection induced strong inflammatory responses in all participants, the inflammatory response was attenuated with pathways associated with antigen processing and presentation less enriched in those with prior exposure to $P$. vivax, suggesting a more 'tolerogenic' immune response in these individuals [30].

In contrast to this work, Rothen et al. found that transcriptional changes post-CHMI via intradermal injection of cryopreserved $P$. falciparum sporozoites were most pronounced on day 5 after inoculation, during the clinically silent liver stage rather than during the blood-stage of infection [38].

\section{Transcriptomic studies in specific cell types}

Whilst the majority of studies examined the immune response from whole blood or PBMCs, some examined transcriptomic changes in other cell types or tissues [26, 33, 39-45]. For example, the work of Muehlenbachs et al. with placental tissue highlighted a previously unappreciated role for B cells in chronic placental malaria [39]; whilst Sullivan et al. compared GEPs of classical and 'atypical' memory B cells obtained from Ugandan children showing the latter demonstrated down-regulation of B cell receptor signalling and apoptosis [43].

\section{Discussion}

GEP is a powerful tool to analyse the immune response to infection. As this review demonstrates, the application of these studies for malaria are wide-ranging, from attempts to dissect the mechanisms of NAI to improving understanding of the interaction between host genotype and infection outcome. However, as a field in its relative infancy, studies are often hypothesis generating with extremely small sample sizes. There is a lack of standardization ranging from methodological (such as sample type, RNA extraction, platform and analysis) to phenotype (including precision in disease context and immune status). This variation means interpreting published data and comparison between studies is challenging. Some of this is unavoidable, however, much could be addressed, for example by implementing standardization in blood sampling, methodological protocols for data generation and analysis with robust significance testing and approaches to confounders, use of ontologies (for example human phenotype and gene ontologies) and expert curation and annotation of data on deposition [46-49].

GEP studies are well placed to examine the mechanisms of NAI and have already helped highlight the role of the innate and early adaptive immune responses [24-26]. However, work has been limited by the lack of an in vitro 
correlate or universally accepted definition of NAI, meaning identifying the immune status of individuals or quantification of immunity is problematic $[6,50]$. In field studies where the timing of infection and parasite burden and dynamics are unknown, and potentially hugely variable between individuals, only limited information can be reliably extrapolated from any GEP changes seen. Most studies assess gene expression from peripheral blood or its components, which does not provide reliable information regarding the transcriptional changes in key organs such as spleen, liver, and bone marrow. In addition, when subjects are recruited at presentation with disease, no baseline comparator data are available to use as a control. Even if a clear difference in GEP were to be reported between individuals with and without NAI, it would be near impossible to distinguish GEP changes associated with parasitaemia from those mediating immunity.

However, there is much potential for the future use of GEP studies, particularly in CHMI studies [51, 52] where the parasite burden can be pre-defined and dynamics of infection closely monitored using highly sensitive qPCR. As these studies are increasingly performed in endemic settings [53-55], there will be growing opportunity to use GEP to understand detailed time-course changes in immune response, particularly at the skin, liver and presymptomatic blood-stage, which to date have been difficult to study in human subjects infected in the field.

\section{Conclusion}

GEP in malaria is a potentially powerful tool, but to date studies have been hypothesis generating with small sample sizes and widely varying methodology. As CHMI studies are increasingly performed in endemic settings, there will be growing opportunity to use GEP to understand detailed time-course changes in host response and understand in greater detail the mechanisms of NAI.

\section{Supplementary information}

Supplementary information accompanies this paper at https://doi. org/10.1186/s12936-019-3035-0.

Additional file 1: Figure S1. Flowchart summarizing identification of GEO datasets and publications.

\section{Abbreviations}

CHMI: controlled human malaria infection; GEO: gene expression omnibus; GEP: gene expression profile; NAl: naturally acquired immunity; PBMC: peripheral blood mononuclear cells.

\section{Acknowledgements}

Not applicable.

\section{Authors' contributions}

$\mathrm{SH}$ conceived the work, analysed the datasets and wrote the manuscript. JM conducted the methodological review of the datasets. HEL, SJD, AVSH, JCK and KM made significant contributions to the conception of the work. JCK substantially revised the manuscript. All authors read and approved the final manuscript.

\section{Funding}

This work was supported by The Wellcome Trust [Grant Number 097940/Z/11/Z to SHH and Wellcome Trust Core Award Grant Number 090532/Z/09/Z]. SHH is a NIHR Academic Clinical Lecturer in Infectious Diseases \& Microbiology at the University of Oxford and Research Fellow at St. Peter's College, University of Oxford. SJD and AVSH are Jenner Investigators, and SJD is also a Lister Institute Research Prize Fellow and a Wellcome Trust Senior Fellow [106917/Z/15/Z]. JCK is a Wellcome Trust Investigator. The funders had no role in the design, collection, analysis, or interpretation of data; in the writing of the manuscript; or in the decision to submit the manuscript for publication.

\section{Availability of data and materials}

Data sharing is not applicable to this article as no datasets were generated or analysed during the current study

Ethics approval and consent to participate

Not applicable.

\section{Consent for publication}

Not applicable.

\section{Competing interests}

The authors declare that they have no competing interests.

\section{Author details}

${ }^{1}$ The Jenner Institute, University of Oxford, Old Road Campus Road Building, Off Roosevelt Drive, Oxford OX3 7DQ, UK. ${ }^{2}$ Department of Infectious Diseases \& Microbiology, Oxford University Hospitals Trust, Oxford, UK. ${ }^{3}$ Wellcome Centre for Human Genetics, University of Oxford, Oxford, UK. ${ }^{4}$ Department of Tropical Medicine, University of Oxford, Oxford, UK.

Received: 15 April 2019 Accepted: 26 November 2019

Published online: 13 December 2019

\section{References}

1. White NJ, Pukrittayakamee S, Hien TT, Faiz MA, Mokuolu OA, Dondorp AM. Malaria. Lancet. 2014;383:723-35.

2. Cockburn IA, Seder RA. Malaria prevention: from immunological concepts to effective vaccines and protective antibodies. Nat Immunol. 2018;19:1199-211.

3. WHO. World malaria report. Geneva: World Health Organization; 2018.

4. Cohen S, Mc Gl, Carrington S. Gamma-globulin and acquired immunity to human malaria. Nature. 1961;192:733-7.

5. Sabchareon A, Burnouf T, Ouattara D, et al. Parasitologic and clinical human response to immunoglobulin administration in falciparum malaria. Am J Trop Med Hyg. 1991;45:297-308.

6. Doolan DL, Dobano C, Baird JK. Acquired immunity to malaria. Clin Microbiol Rev. 2009;22:13-36.

7. Conesa A, Mortazavi A. The common ground of genomics and systems biology. BMC Syst Biol. 2014;8(Suppl 2):S1.

8. Schulze A, Downward J. Navigating gene expression using microarraysa technology review. Nat Cell Biol. 2001;3:E190-5.

9. Wang Z, Gerstein M, Snyder M. RNA-Seq: a revolutionary tool for transcriptomics. Nat Rev Genet. 2009;10:57-63.

10. Papalexi E, Satija R. Single-cell RNA sequencing to explore immune cell heterogeneity. Nat Rev Immunol. 2018;18:35-45.

11. Querec TD, Akondy RS, Lee EK, Cao W, Nakaya HI, Teuwen D, et al. Systems biology approach predicts immunogenicity of the yellow fever vaccine in humans. Nat Immunol. 2009;10:116-25.

12. Six A, Bellier B, Thomas-Vaslin V, Klatzmann D. Systems biology in vaccine design. Microb Biotechnol. 2012;5:295-304.

13. Rappuoli R, Aderem A. A 2020 vision for vaccines against HIV, tuberculosis and malaria. Nature. 2011;473:463-9. 
14. Hogan CM, Hammer SM. Host determinants in HIV infection and disease. Part 1: cellular and humoral immune responses. Ann Intern Med. 2001:134:761-76.

15. Blohmke CJ, Darton TC, Jones C, Suarez NM, Waddington CS, Angus B, et al. Interferon-driven alterations of the host's amino acid metabolism in the pathogenesis of typhoid fever. J Exp Med. 2016;213:1061-77.

16. Wong HR. Clinical review: sepsis and septic shock - the potential of gene arrays. Crit Care. 2012;16:204.

17. Anderson ST, Kaforou M, Brent AJ, Wright VJ, Banwell CM, Chagaluka G, et al. Diagnosis of childhood tuberculosis and host RNA expression in Africa. N Engl J Med. 2014;370:1712-23.

18. Mejias A, Ramilo O. Transcriptional profiling in infectious diseases: ready for prime time? J Infect. 2014;68(Suppl 1):S94-9.

19. Berry MP, Graham CM, McNab FW, Xu Z, Bloch SA, Oni T, et al. An interferon-inducible neutrophil-driven blood transcriptional signature in human tuberculosis. Nature. 2010;466:973-7.

20. Westermann AJ, Forstner KU, Amman F, Barquist L, Chao Y, Schulte LN, et al. Dual RNA-seq unveils noncoding RNA functions in host-pathogen interactions. Nature. 2016;529:496-501.

21. Cummings CA, Relman DA. Using DNA microarrays to study hostmicrobe interactions. Emerg Infect Dis. 2000;6:513-25.

22. Dunachie $\mathrm{S}$, Hill AV, Fletcher HA. Profiling the host response to malaria vaccination and malaria challenge. Vaccine. 2015;33:5316-20.

23. Griffiths MJ, Shafi MJ, Popper SJ, Hemingway CA, Kortok MM, Wathen A, et al. Genomewide analysis of the host response to malaria in Kenyan children. J Infect Dis. 2005;191:1599-611.

24. Krupka M, Seydel K, Feintuch CM, Yee K, Kim R, Lin CY, et al. Mild Plasmodium falciparum malaria following an episode of severe malaria is associated with induction of the interferon pathway in Malawian children. Infect Immun. 2012;80:1150-5.

25. Tran TM, Jones MB, Ongoiba A, Bijker EM, Schats R, Venepally P, et al. Transcriptomic evidence for modulation of host inflammatory responses during febrile Plasmodium falciparum malaria. Sci Rep. 2016;6:31291.

26. Jagannathan P, Kim CC, Greenhouse B, Nankya F, Bowen K, Eccles-James I, et al. Loss and dysfunction of Vdelta2(+) gammadelta T cells are associated with clinical tolerance to malaria. Sci Transl Med. 2014;6:251 ra117.

27. Barrett T, Wilhite SE, Ledoux P, Evangelista C, Kim IF, Tomashevsky M, et al. NCBI GEO: archive for functional genomics data sets-update. Nucleic Acids Res. 2013;41:D991-5.

28. Rojas-Pena ML, Vallejo A, Herrera S, Gibson G, Arevalo-Herrera M. Transcription profiling of malaria-naive and semi-immune Colombian volunteers in a Plasmodium vivax sporozoite challenge. PLoS Negl Trop Dis. 2015;9:e0003978.

29. Ockenhouse CF, Hu WC, Kester KE, Cummings JF, Stewart A, Heppner $D G$, et al. Common and divergent immune response signaling pathways discovered in peripheral blood mononuclear cell gene expression patterns in presymptomatic and clinically apparent malaria. Infect Immun. 2006;74:5561-73

30. Vallejo AF, Read RC, Arevalo-Herrera M, Herrera S, Elliott T, Polak ME. Malaria systems immunology: Plasmodium vivax induces tolerance during primary infection through dysregulation of neutrophils and dendritic cells. J Infect. 2018;77:440-7.

31. Boldt ABW, van Tong H, Grobusch MP, Kalmbach Y, Dzeing Ella A, Kombila $M$, et al. The blood transcriptome of childhood malaria. EBioMedicine. 2019;40:614-25.

32. Subramaniam KS, Spaulding E, Ivan E, Mutimura E, Kim RS, Liu X, et al. The T-cell inhibitory molecule Butyrophilin-Like 2 is up-regulated in mild Plasmodium falciparum infection and is protective during experimental cerebral malaria. J Infect Dis. 2015;212:1322-31.

33. Quin JE, Bujila I, Cherif M, Sanou GS, Qu Y, Vafa Homann M, et al. Major transcriptional changes observed in the Fulani, an ethnic group less susceptible to malaria. Elife. 2017;6:e29156.

34. Franklin BS, Parroche P, Ataide MA, Lauw F, Ropert C, de Oliveira RB, et al Malaria primes the innate immune response due to interferon-gamma induced enhancement of toll-like receptor expression and function. Proc Natl Acad Sci USA. 2009;106:5789-94.

35. Sharma S, DeOliveira RB, Kalantari P, Parroche P, Goutagny N, Jiang Z, et al. Innate immune recognition of an AT-rich stem-loop DNA motif in the Plasmodium falciparum genome. Immunity. 2011;35:194-207.
36. Idaghdour Y, Quinlan J, Goulet JP, Berghout J, Gbeha E, Bruat V, et al. Evidence for additive and interaction effects of host genotype and infection in malaria. Proc Natl Acad Sci USA. 2012;109:16786-93.

37. Gardinassi LG, Arevalo-Herrera M, Herrera S, Cordy RJ, Tran V, Smith MR, et al. Integrative metabolomics and transcriptomics signatures of clinical tolerance to Plasmodium vivax reveal activation of innate cell immunity and T cell signaling. Redox Biol. 2018;17:158-70.

38. Rothen J, Murie C, Carnes J, Anupama A, Abdulla S, Chemba M, et al. Whole blood transcriptome changes following controlled human malaria infection in malaria pre-exposed volunteers correlate with parasite prepatent period. PLoS ONE. 2018;13:e0199392.

39. Muehlenbachs A, Fried M, Lachowitzer J, Mutabingwa TK, Duffy PE. Genome-wide expression analysis of placental malaria reveals features of lymphoid neogenesis during chronic infection. J Immunol. 2007;179:557-65.

40. Burel JG, Apte SH, Groves PL, McCarthy JS, Doolan DL. Polyfunctional and IFN-gamma monofunctional human CD4+ T cell populations are molecularly distinct. JCI Insight. 2017;2:e87499.

41. Jaijyan DK, Singh H, Singh AP. A sporozoite- and liver stage-expressed tryptophan-rich protein plays an auxiliary role in Plasmodium liver stage development and is a potential vaccine candidate. J Biol Chem. 2015;290:19496-511.

42. Terkawi MA, Takano R, Furukawa A, Murakoshi F, Kato K. Involvement of beta-defensin 130 (DEFB130) in the macrophage microbicidal mechanisms for killing Plasmodium falciparum. Sci Rep. 2017;7:41772.

43. Sullivan RT, Kim CC, Fontana MF, Feeney ME, Jagannathan P, Boyle MJ, et al. FCRL 5 delineates functionally impaired memory B cells associated with Plasmodium falciparum exposure. PLoS Pathog. 2015;11:e1004894.

44. Terkawi MA, Takano R, Kato K. Differential gene expression profile of human neutrophils cultured with Plasmodium falciparum-parasitized erythrocytes. J Immunol Res. 2018;2018:6709424.

45. Portugal S, Tipton CM, Sohn H, Kone Y, Wang J, Li S, et al. Malaria-associated atypical memory $B$ cells exhibit markedly reduced $B$ cell receptor signaling and effector function. Elife. 2015;4:e07218.

46. Bammler T, Beyer RP, Bhattacharya S, Boorman GA, Boyles A, Bradford BU, et al. Standardizing global gene expression analysis between laboratories and across platforms. Nat Methods. 2005;2:351-6.

47. Conesa A, Madrigal P, Tarazona S, Gomez-Cabrero D, Cervera A, McPherson A, et al. A survey of best practices for RNA-seq data analysis. Genome Biol. 2016;17:13

48. Chowdhury HA, Bhattacharyya DK, Kalita JK. (Differential) co-expression analysis of gene expression: a survey of best practices. IEEE/ACM Trans Comput Biol Bioinform. 2019. https://doi.org/10.1109/TCBB.2019.2893170 (Epub ahead of print).

49. Rung J, Brazma A. Reuse of public genome-wide gene expression data. Nat Rev Genet. 2013:14:89-99.

50. Langhorne J, Ndungu FM, Sponaas AM, Marsh K. Immunity to malaria: more questions than answers. Nat Immunol. 2008;9:725-32.

51. Barton AJ, Hill J, Pollard AJ, Blohmke CJ. Transcriptomics in human challenge models. Front Immunol. 2017;8:1839.

52. Stanisic DI, McCarthy JS, Good MF. Controlled human malaria infection: applications, advances, and challenges. Infect Immun. 2018;86:e00479.

53. Lell B, Mordmuller B, Dejon Agobe JC, Honkpehedji J, Zinsou J, Mengue $\mathrm{JB}$, et al. Impact of sickle cell trait and naturally acquired immunity on uncomplicated malaria after controlled human malaria infection in adults in Gabon. Am J Trop Med Hyg. 2018;98:508-15.

54. Hodgson SH, Juma E, Salim A, Magiri C, Kimani D, Njenga D, et al. Evaluating controlled human malaria infection in Kenyan adults with varying degrees of prior exposure to Plasmodium falciparum using sporozoites administered by intramuscular injection. Front Microbiol. 2014;5:686.

55. Shekalaghe S, Rutaihwa M, Billingsley PF, Chemba M, Daubenberger CA, James ER, et al. Controlled human malaria infection of Tanzanians by intradermal injection of aseptic, purified, cryopreserved Plasmodium falciparum sporozoites. Am J Trop Med Hyg. 2014;91:471-80.

\section{Publisher's Note}

Springer Nature remains neutral with regard to jurisdictional claims in published maps and institutional affiliations. 